KEK Preprint 2001-164

\title{
Quantum Tunneling and Caustics under Inverse Square Potential
}

\author{
Hitoshi Miyazaki* and Izumi Tsutsui ${ }^{\dagger}$ \\ Institute of Particle and Nuclear Studies \\ High Energy Accelerator Research Organization (KEK) \\ Tsukuba 305-0801 \\ Japan
}

\begin{abstract}
We examine the quantization of a harmonic oscillator with inverse square potential $V(x)=\left(m \omega^{2} / 2\right) x^{2}+g / x^{2}$ on the line $-\infty<x<\infty$. We find that, for $0<g<3 \hbar^{2} /(8 m)$, the system admits a $U(2)$ family of inequivalent quantizations allowing for quantum tunneling through the infinite potential barrier at $x=0$. These are a generalization of the conventional quantization applied to the Calogero model in which no quantum tunneling is allowed. The tunneling renders the classical caustics which arise under the potential anomalous at the quantum level, leading to the possibility of copying the profile of an arbitrary state from one side $x>0$, say, to the other $x<0$.
\end{abstract}

PACS codes: 03.65.-w, 73.63.-b, 02.30.-f

* email: miyazaki@post.kek.jp

$\dagger$ email: izumi.tsutsui@kek.jp 


\section{Introduction}

In some dynamical systems there occurs a peculiar phenomenon that classical trajectories focus on one single point after a lapse of a certain time irrespective of their initial conditions. This occurs typically in a harmonic oscillator, where the oscillator returns to the initial position periodically whatever its initial velocity is. This phenomenon underlies the classical caustics of geometrical optics, whose quantum version has also been studied earlier $[1,2]$. Since the phenomenon is genuinely classical, one is tempted to ask if any substantial change occurs in the caustics at the quantum level. A path-integral analysis $[3,4]$ indicates, however, that for quadratic systems the focusing phenomenon remains essentially unchanged after quantization - it arises as a recurrence of the initial profile of probability distributions, accompanied by certain quantum effects [5].

The caustics phenomenon remains to be seen, at least classically, even when an inverse square potential is added to the harmonic oscillator. Indeed, the Hamiltonian,

$$
H(p, x)=\frac{1}{2 m} p^{2}+\frac{m \omega^{2}}{2} x^{2}+g \frac{1}{x^{2}},
$$

on the line $-\infty<x<\infty$ admits classical solutions which exhibit periodicity for positive strength $g>0$, implying that caustics still occur despite that the system is now nonquadratic. This system is in fact the quantum solvable model considered by Calogero [6] and, like in quadratic systems, has been argued to exhibit corresponding caustics at the quantum level [7]. The argument is based on the conventional quantization of the system (1) used for the Calogero model which assumes no probability flow to pass the singular (infinite) barrier at $x=0$. Because of this prohibition of quantum tunneling, the system does not reduce to the harmonic oscillator for $g \rightarrow 0$ as one naïvely expects.

On the other hand, it has been known in the mathematical literature that systems with singularity such as the one mentioned above may have inequivalent quantizations due to the arbitrariness of the boundary (or connection) condition at the singularity (see, e.g., $[8,9]$ for systems on the half line, and $[10,11]$ for those with point interaction). In this paper we examine the quantization of the system (1) from this viewpoint and point out that, for $g$ in the range,

$$
0<g<\frac{3 \hbar^{2}}{8 m}
$$

the system admits a $U(2)$ family of inequivalent quantizations. In contrast to the conventionl one, most of these quantizations permit quantum tunneling through the infinite barrier at $x=0$. In particular, we find that there is a distinguished quantization possessing a smooth limit to the harmonic oscillator for $g \rightarrow 0$. Remarkably, in this quantization 
the classical picture of the caustics acquires a drastic change in the quantum regime: the focusing occurs in two points, rather than one. We show that this anomalous phenomenon of quantum caustics may be used to copy the profile of an arbitrary state from one side $x>0$, say, to the other $x<0$.

\section{Classical caustics and quantum states}

Before delving into the discussion of quantization of the system (1), let us quickly recall how the phenomenon of the classical caustics can be observed. Let $H(p, x)=E$ be the energy of the solution we are looking for. With $p=m \dot{x}$ the constant energy equation can readily be integrated to give the classical solution,

$$
x(t)= \pm\left\{\sqrt{\frac{E^{2}-2 g m \omega^{2}}{\left(m \omega^{2}\right)^{2}}} \sin \left(2 \omega\left(t+t_{0}\right)\right)+\frac{E}{m \omega^{2}}\right\}^{1 / 2} .
$$

The integration constant $t_{0}$ and the energy $E$ are altered according to the initial position $x\left(t_{i}\right)$ and velocity $\dot{x}\left(t_{i}\right)$ chosen arbitrarily, but for any choice the particle returns to the original position $x\left(t_{i}+T\right)=x\left(t_{i}\right)$ for $T=k \pi / \omega$ with integer $k$. These are the classical caustics appearing in the system (1).

The quantum system corresponding to (1), too, admits exact solutions of eigenstates for the Schrödinger equation. Although the procedure to obtain the solutions has been given in various references (see, e.g., $[6,12]$ ), we shall present here a fuller treatment paying a special attention to the boundary condition at $x=0$. To proceed, we remove the singular point $x=0$ from the system to define our Hilbert space as $\mathcal{H}=L^{2}(\mathbb{R} \backslash\{0\})$. The boundary condition is then considered at the limiting points $x \rightarrow \pm 0$. For the Hamiltonian operator $\widehat{H}=H(-i \hbar d / d x, x)$ the Schrödinger equation for energy eigenstates reads

$$
\widehat{H} \psi_{n}(x)=\left(-\frac{\hbar^{2}}{2 m} \frac{d^{2}}{d x^{2}}+\frac{m \omega^{2}}{2} x^{2}+g \frac{1}{x^{2}}\right) \psi_{n}(x)=E_{n} \psi_{n}(x) .
$$

For the moment we only consider the positive half line $\mathbb{R}_{+}=\{x>0\}$, but the negative half $\mathbb{R}_{-}=\{x<0\}$ can be handled analogously using the solutions on $\mathbb{R}_{+}$. If we set

$$
\psi_{n}(x)=y^{a+1 / 2} e^{-y^{2} / 2} f_{n}\left(y^{2}\right), \quad y=\sqrt{\frac{m \omega}{\hbar}} x,
$$

and choose

$$
a=\frac{1}{2} \sqrt{1+\frac{8 m g}{\hbar^{2}}}
$$


then the Schrödinger equation (4) becomes

$$
z \frac{d^{2} f_{n}}{d z^{2}}(z)+(a+1-z) \frac{d f_{n}}{d z}(z)-\frac{1}{2}\left(a+1-\lambda_{n}\right) f_{n}(z)=0, \quad \lambda_{n}=\frac{E_{n}}{\hbar \omega},
$$

under the variable $z=y^{2}$. This is the confluent hypergeometric differential equation $z f^{\prime \prime}(z)+(\gamma-z) f^{\prime}(z)-\alpha f(z)=0$, whose two independent solutions are, for $\gamma \neq$ integer, given by $f(z)=F(\alpha, \gamma ; z)$ and $z^{1-\gamma} F(\alpha-\gamma+1,2-\gamma ; z)$ with $F(\alpha, \gamma ; z)$ being the confluent hypergeometric function. Thus the two independent solutions for the Schrödinger equation (4) are

$$
\begin{array}{ll}
\phi_{n}^{(1)}(x):=y^{c_{1}-1 / 2} e^{-y^{2} / 2} F\left(\frac{c_{1}-\lambda_{n}}{2}, c_{1} ; y^{2}\right), & c_{1}=1+a, \\
\phi_{n}^{(2)}(x):=y^{c_{2}-1 / 2} e^{-y^{2} / 2} F\left(\frac{c_{2}-\lambda_{n}}{2}, c_{2} ; y^{2}\right), & c_{2}=1-a .
\end{array}
$$

The general solution $\psi_{n}(x)$ of (4) is given by a linear combination of these two, but since the combination may differ on the two sides $\mathbb{R}_{+}$and $\mathbb{R}_{-}$, we have

$$
\psi_{n}(x)=\left[N_{\mathrm{R}}^{(1)} \phi_{n}^{(1)}(|x|)+N_{\mathrm{R}}^{(2)} \phi_{n}^{(2)}(|x|)\right] \Theta(x)+\left[N_{\mathrm{L}}^{(1)} \phi_{n}^{(1)}(|x|)+N_{\mathrm{L}}^{(2)} \phi_{n}^{(2)}(|x|)\right] \Theta(-x),
$$

where $N_{\mathrm{R}}^{(s)}$ and $N_{\mathrm{L}}^{(s)}$ are arbitrary constants and $\Theta(x)$ is the Heaviside step function.

At this point let us examine the normalizability (square integrability) of the solutions (8). First, since $c_{1}>3 / 2$ and $c_{2}<1 / 2$, we observe that as $x \rightarrow 0$ the solution $\phi_{n}^{(1)}$ approaches zero while $\phi_{n}^{(2)}$ diverges. From $\int_{0}^{\epsilon} d x\left|\phi_{n}^{(2)}(x)\right|^{2} \simeq \epsilon^{2 c_{2}}$ for a small $\epsilon$, we realize that $\phi_{n}^{(2)}$ can be normalizable for $c_{2}>0$. This is the case if the coupling constant $g$ satisfies (2), and we confine ourselves to this case hereafter. (For the normalizability $g$ may be nonpositive, but for our consideration of quantum tunneling and caustics we assume $g>0$.) Note that (2) implies $3 / 2<c_{1}<2$ and $0<c_{2}<1 / 2$, and this allows us to disregard the case $\gamma=$ integer in considering the solution of (7). Once the two independent solutions are admitted from the behaviour near $x=0$, then the normalizability is ensured if the solution vanishes sufficiently fast at the infinity $x= \pm \infty$. From the asymptotic behaviour of the confluent hypergeometric function,

$$
F(\alpha, \gamma ; z) \sim \frac{\Gamma(\gamma)}{\Gamma(\alpha)} e^{z} z^{\alpha-\gamma}, \quad \text { as } \quad|z| \rightarrow \infty
$$

we find that the normalizability of the solutions (8) requires

$$
\frac{N_{\mathrm{R}}^{(1)}}{N_{\mathrm{R}}^{(2)}}=\frac{N_{\mathrm{L}}^{(1)}}{N_{\mathrm{L}}^{(2)}}=-\frac{\Gamma\left(\left(c_{1}-\lambda_{n}\right) / 2\right)}{\Gamma\left(\left(c_{2}-\lambda_{n}\right) / 2\right)} \frac{\Gamma\left(c_{2}\right)}{\Gamma\left(c_{1}\right)}
$$


Another condition to be imposed on the solutions is the boundary condition at the singular point $x=0$. This is needed to ensure the continuity of the probability current at $x=0$, which is equivalent to ensuring that the Hamiltonian $\widehat{H}$ be self-adjoint. It is known $[8,9]$ that, in the presence of singularity, there can exist (at most) a $U(2)$ family of self-adjoint Hamiltonians specified by corresponding boundary conditions. By means of the Wronskian $W[\psi, \varphi](x)=(\psi(d \varphi / d x)-(d \psi / d x) \varphi)(x)$, which is finite even if the wavefunctions $\psi(x), \varphi(x)$ may be divergent at the singularity, the boundary conditions are presented as follows $[13,14]$ (see $[15,16]$ for the conditions on the line). Let $\varphi_{1}, \varphi_{2}$ be two independent, real zero modes,

$$
\widehat{H} \varphi_{1}(x)=\widehat{H} \varphi_{2}(x)=0, \quad W\left[\varphi_{1}, \varphi_{2}\right](x)=1
$$

Given a state $\psi$ which is normalizable, we introduce the complex column vectors,

$$
\Psi=\left(\begin{array}{c}
W\left[\psi, \varphi_{1}\right]_{+0} \\
W\left[\psi, \varphi_{1}\right]_{-0}
\end{array}\right), \quad \Psi^{\prime}=\left(\begin{array}{c}
W\left[\psi, \varphi_{2}\right]_{+0} \\
-W\left[\psi, \varphi_{2}\right]_{-0}
\end{array}\right)
$$

defined from the boundary values $W[\psi, \varphi]_{ \pm 0}:=\lim _{x \rightarrow \pm 0} W[\psi, \varphi](x)$. The boundary condition for $\psi \in \mathcal{H}$ is then given by

$$
(U-I) \Psi+i L_{0}(U+I) \Psi^{\prime}=0
$$

where $U$ is a $U(2)$ matrix, $I$ is the identity matrix, and $L_{0}$ is a constant with dimension of length. This way a self-adjoint Hamiltonian is specified uniquely by the matrix $U$, which may hence be called the 'characteristic matrix'.

In our case, we label $n=n_{0}$ for which $\lambda_{n_{0}}=0$ in (8) and set

$$
\begin{aligned}
\varphi_{1}(x) & :=\sqrt{\frac{\hbar}{m \omega}} \phi_{n_{0}}^{(1)}(|x|)[\Theta(x)-\Theta(-x)], \\
\varphi_{2}(x) & :=\frac{1}{c_{2}-c_{1}} \phi_{n_{0}}^{(2)}(|x|),
\end{aligned}
$$

so that (12) is fulfilled. Since $F(\alpha, \gamma ; z)=1+\mathcal{O}(z)$ as $z \rightarrow 0$, the boundary vectors (13) for the solution $\psi_{n}$ in (9) turn out to be

$$
\Psi=\left(c_{1}-c_{2}\right)\left(\begin{array}{c}
N_{\mathrm{R}}^{(2)} \\
N_{\mathrm{L}}^{(2)}
\end{array}\right), \quad \Psi^{\prime}=\sqrt{\frac{m \omega}{\hbar}}\left(\begin{array}{c}
N_{\mathrm{R}}^{(1)} \\
N_{\mathrm{L}}^{(1)}
\end{array}\right) .
$$

The relations (11) and (16) then imply that the vector $\Psi^{\prime}$ is proportional to $\Psi$, and hence there exists a constant $\xi$ such that $\Psi^{\prime}=\xi \Psi$. Thus the boundary condition (14) is now

$$
\left[(U-I)+i L_{0} \xi(U+I)\right] \Psi=0
$$


and, in order to obtain a non-trivial vector $\Psi$, we need

$$
\operatorname{det}\left|U-I+i L_{0} \xi(U+I)\right|=\operatorname{det}\left|D-I+i L_{0} \xi(D+I)\right|=0
$$

where we have decomposed $U \in U(2)$ as $U=V^{-1} D V$ using some $S U(2)$ matrix $V$ and a diagonal matrix $D$. In terms of the parameterization,

$$
D=\left(\begin{array}{cc}
e^{i \theta_{+}} & 0 \\
0 & e^{i \theta_{-}}
\end{array}\right)
$$

with $\theta_{ \pm} \in[0,2 \pi)$, we find that (18) is satisfied if

$$
\xi=-\frac{1}{L_{+}} \quad \text { or } \quad-\frac{1}{L_{-}}, \quad L_{ \pm}=L_{0} \cot \left(\frac{\theta_{ \pm}}{2}\right) .
$$

Substituting this back to (11), we obtain

$$
\frac{\Gamma\left(\left(c_{1}-\lambda_{n}\right) / 2\right)}{\Gamma\left(\left(c_{2}-\lambda_{n}\right) / 2\right)} \frac{\Gamma\left(c_{2}\right)}{\Gamma\left(c_{1}\right)}=\sqrt{\frac{\hbar}{m \omega}} \frac{c_{1}-c_{2}}{L_{+}} \text {or } \sqrt{\frac{\hbar}{m \omega}} \frac{c_{1}-c_{2}}{L_{-}}
$$

from which we determine the energy spectrum $\left\{E_{n}=\lambda_{n} \hbar \omega\right\}$ of our system. The ratios $N_{\mathrm{R}}^{(1)} / N_{\mathrm{R}}^{(2)}$ and $N_{\mathrm{L}}^{(1)} / N_{\mathrm{L}}^{(2)}$ are determined once either $L_{+}$or $L_{-}$is chosen. Our result shows that the system permits two distinct series of eigenstates generically, one specified by $L_{+}$ and the other by $L_{-}$, and this illustrates the fact that any one dimensional system which admits a $U(2)$ family of self-adjoint Hamiltonians possesses a spectral family parametrized by two angle parameters $[15,16]$.

We shall mention a few cases where the spectrum $\left\{E_{n}\right\}$ can be obtained explicitly. First, if $\left(\theta_{+}, \theta_{-}\right)=(0,0)$, then $1 / L_{ \pm}=0$ and hence $(21)$ is fulfilled by those $\lambda_{n}$ for which the Gamma function in the denominator has poles. This leads to $E_{n}=\left(2 n+c_{2}\right) \hbar \omega$ and the eigenstate given by $\phi_{n}^{(2)}(|x|)$ either on $\mathbb{R}_{+}$or $\mathbb{R}_{-}$(hence each level is doubly degenerated). Similarly, if $\left(\theta_{+}, \theta_{-}\right)=(\pi, \pi)$, then $L_{ \pm}=0$ and one obtains $E_{n}=\left(2 n+c_{1}\right) \hbar \omega$ and the eigenstate $\phi_{n}^{(1)}(|x|)$ which is also doubly degenerated. This is the case (which amounts to the choice $U=-I$ ) that has been considered conventionally in the treatment of the system (4) since the early days of Calogero [6].

On the other hand, if $\left(\theta_{+}, \theta_{-}\right)=(0, \pi)$, then $1 / L_{+}=0=L_{-}$, which means that there occurs two series of eigenstates, one with $N_{\mathrm{R}}^{(2)}=N_{\mathrm{L}}^{(2)}=0$ and the other with $N_{\mathrm{R}}^{(1)}=N_{\mathrm{L}}^{(1)}=0$, whose eigenvalues are

$$
E_{n}^{(1)}=\left(2 n+c_{1}\right) \hbar \omega, \quad E_{n}^{(2)}=\left(2 n+c_{2}\right) \hbar \omega
$$


respectively. In particular, in the limit $g \rightarrow 0$ we have $c_{1} \rightarrow 3 / 2$ and $c_{2} \rightarrow 1 / 2$, which shows that our system recovers the spectrum of a harmonic oscillator. A complete reduction to the harmonic oscillator system is realized by choosing $U=\sigma_{1}$ (where $\left\{\sigma_{i}\right\}$ are Pauli matrices), which is obtained by setting $V=e^{i \pi \sigma_{2} / 4}$ as well as $\left(\theta_{+}, \theta_{-}\right)=(0, \pi)$. For this choice, the boundary condition (17) requires $N_{\mathrm{R}}^{(1)}=-N_{\mathrm{L}}^{(1)}, N_{\mathrm{R}}^{(2)}=N_{\mathrm{L}}^{(2)}$ and hence the two series of eigenstates in (9) are found to be

$$
\begin{aligned}
& \psi_{n}^{(1)}(x):=N^{(1)} \phi_{n}^{(1)}(|x|)[\Theta(x)-\Theta(-x)], \\
& \psi_{n}^{(2)}(x):=N^{(2)} \phi_{n}^{(2)}(|x|),
\end{aligned}
$$

for $n=0,1,2 \ldots$, where $N^{(s)}=\left[\sqrt{m w / \hbar} \Gamma\left(n+c_{s}\right) /\left\{\left(\Gamma\left(c_{s}\right)\right)^{2} n !\right\}\right]^{1 / 2}$ for $s=1,2$ are normalization constants determined so that $\int_{-\infty}^{\infty} d x\left|\psi_{n}^{(s)}(x)\right|^{2}=1$. The eigenfunctions (23) reduce exactly to those of the harmonic oscillator in the limit $g \rightarrow 0$, that is, $\psi_{n}^{(1)}$ reduces to $e^{-y^{2} / 2} H_{2 n+1}(y)$ and $\psi_{n}^{(2)}$ to $e^{-y^{2} / 2} H_{2 n}(y)$ where $H_{n}$ is the Hermite polynomial of degree $n$. This in turn implies that, for other $U$, the system does not lead to a harmonic oscillator in the limit, which suggests that our system with finite $g$ may be regarded, effectively, as a system that possesses a singular point interaction at $x=0$ which is hidden in the singularity of the potential. Under regular potentials, point interactions are known to admit a $U(2)$ family of boundary conditions at the singularity, in which $U=\sigma_{1}$ provides the boundary condition for the 'free point', namely, no interaction there [11]. The fact that the smooth limit $g \rightarrow 0$ to the harmonic oscillator is gained at $U=\sigma_{1}$ suggests that the above effective picture for the $U(2)$ family works also for singular potentials. We also mention that the case $U=\sigma_{1}$ corresponds to the quantization discussed in ref.[12] which pointed out that the conventional quantization $U=-I$ cannot be a perturbed harmonic oscillator because of the too severe physical conditions it presupposes.

\section{Quantum caustics and its anomaly}

Now that we have unconventional but perfectly admissible eigenstates arising under the boundary conditions specified by $U$, we next examine how the caustics appear at the quantum level. Before this, however, let us consider the possibility of quantum tunneling though the barrier of the potential at $x=0$. In order to make our discussions clear and simple, we consider only the case $U=\sigma_{1}$ where the eigenstates are given by (23). To investigate whether or not tunneling phenomena occurs, we simply evaluate the probability current $j(+0)(=j(-0))$ for a given arbitrary state $\psi$. Since $(23)$ gives our complete basis, we expand it as $\psi(x)=\sum_{n}\left(c_{n}^{(1)} \psi_{n}^{(1)}(x)+c_{n}^{(2)} \psi_{n}^{(2)}(x)\right)$. Then we find

$$
j(+0):=\frac{\hbar}{2 i m} W\left[\psi^{*}, \psi\right]_{+0}=\frac{i a \hbar}{m} \sum_{n, l}\left\{\left(c_{n}^{(1)}\right)^{*} c_{l}^{(2)}-\left(c_{n}^{(2)}\right)^{*} c_{l}^{(1)}\right\},
$$


which shows that, since $s \neq 0$ for $g>0$, the probability current does flow through the barrier $x=0$. Note that $j(+0) \neq 0$ is realized for states $\psi$ consisting of both type of eigenstates $\psi_{n}^{(1)}$ and $\psi_{l}^{(2)}$, and this is made possible only for $g$ satisfying (2) and further for (generic) $U$, such as the one $U=\sigma_{1}$ we are considering, under which the two type of eigenstates appear. If $g \geq 3 \hbar^{2} / 8$, or else if $U$ is diagonal $U=D$ like the conventional choice $U=-I$, we always have $j(+0)=0$, disconnecting the right and left half lines, $x>0$ and $x<0$, physically.

Once the quantum tunneling is allowed, then the classical picture of caustics, which occur in the half lines independently, is no longer viable, and one is curious what in fact will happen quantum mechanically. To investigate this, we calculate the transition amplitude, the Feynman kernel $K\left(x_{f}, t_{f} ; x_{i}, t_{i}\right)$, from the initial state of the particle staying at $x=x_{i}$ at $t=t_{i}$ to the final state staying at $x=x_{f}$ at $t=t_{f}$. In our case (23), a straightforward computation (see Appendix) yields that for $T:=t_{f}-t_{i} \neq k \pi / \omega$ with $k=0,1,2, \ldots$,

$$
\begin{aligned}
K\left(x_{f}, t_{f} ;\right. & \left.x_{i}, t_{i}\right)=\frac{m \omega}{2 i \hbar \sin (\omega T)}\left(\left|x_{f} x_{i}\right|\right)^{1 / 2} \exp \left(\frac{i}{2} \frac{m \omega}{\hbar} \frac{\cos (\omega T)}{\sin (\omega T)}\left(x_{f}^{2}+x_{i}^{2}\right)\right) \\
\times & {\left[\Theta\left(x_{f} x_{i}\right)\left\{I_{a}\left(\frac{m \omega}{i \hbar} \frac{\left|x_{f} x_{i}\right|}{\sin (\omega T)}\right)+I_{-a}\left(\frac{m \omega}{i \hbar} \frac{\left|x_{f} x_{i}\right|}{\sin (\omega T)}\right)\right\}\right.} \\
+ & \left.\Theta\left(-x_{f} x_{i}\right)\left\{-I_{a}\left(\frac{m \omega}{i \hbar} \frac{\left|x_{f} x_{i}\right|}{\sin (\omega T)}\right)+I_{-a}\left(\frac{m \omega}{i \hbar} \frac{\left|x_{f} x_{i}\right|}{\sin (\omega T)}\right)\right\}\right],
\end{aligned}
$$

where $I_{\nu}(z)$ is the modified Bessel function and $a$ is related to $g$ by (6). The last two terms with the factor $\Theta\left(-x_{f} x_{i}\right)$ represent the transition allowed by the quantum tunneling. One can readily check that the Feynman kernel (25) reduces to that of a harmonic oscillator in the limit $g \rightarrow 0$.

On the other hands, for $T=k \pi / \omega$, we find

$$
K\left(x_{f}, t_{f} ; x_{i}, t_{i}\right)=(-1)^{k} \cos (a k \pi) \delta\left(x_{f}-x_{i}\right)+i(-1)^{k} \sin (a k \pi) \delta\left(x_{f}+x_{i}\right) .
$$

The term containing $\delta\left(x_{f}-x_{i}\right)$ represents the quantum counterpart of the classical caustics, whereas the term containing $\delta\left(x_{f}+x_{i}\right)$ represents extra caustics that arise only at the quantum level through the tunneling effect. We emphasize that the appearance of the anomalous quantum caustics is crucial to achieve the smooth reduction to the harmonic oscillator, since $g \rightarrow 0$ implies $a \rightarrow 1 / 2$ and hence the two terms contribute to the caustics of the harmonic oscillator alternately.

In passing we note that the other limit $a \rightarrow 1$ is also smooth, because then the kernel, (25) or (26), becomes the usual one [17] (since, for (25) the last two terms with 

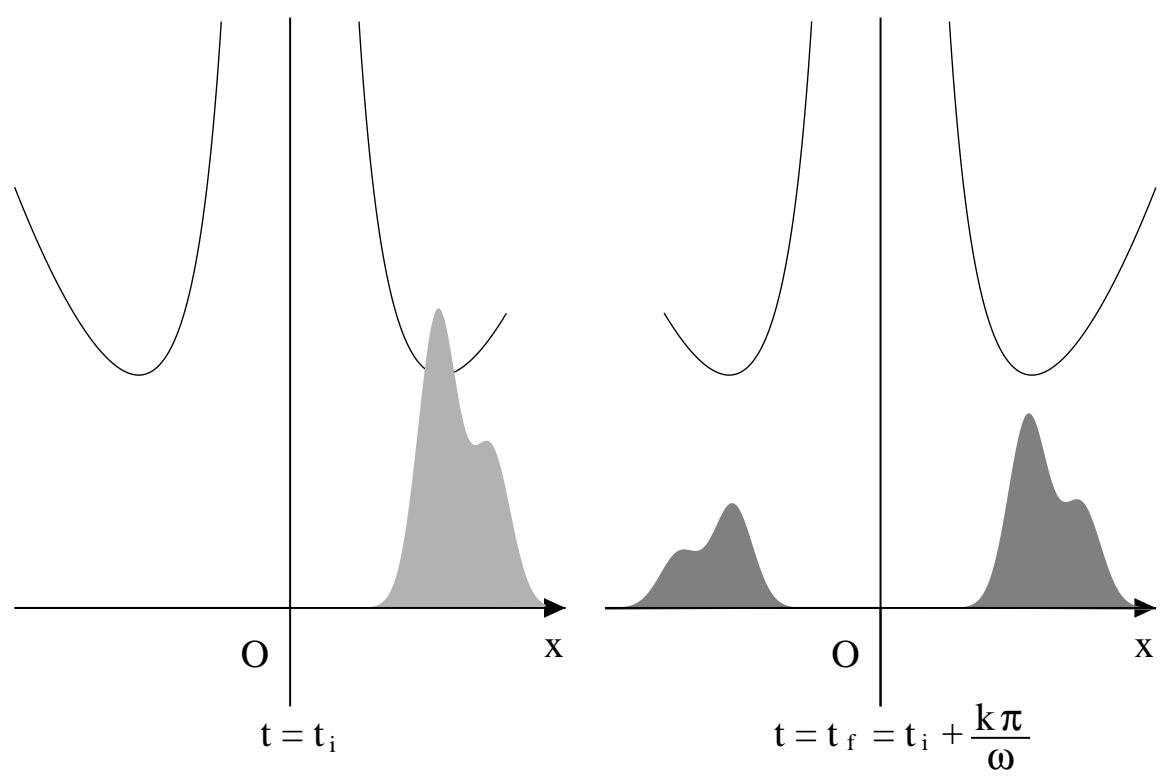

Figure 1. Process of quantum copy through the caustics anomaly. At every period $T=k \pi / \omega$, a mirror image of the original profile on $x>0$ emerges on the other side $x<0$. The relative size of the mirror image depends on $a$ and $k$.

$\Theta\left(-x_{f} x_{i}\right)$ cancel with each other, whereas for (26) we only get $\left.\delta\left(x_{f}-x_{i}\right)\right)$ obtained under the conventional quantization. This is due to the fact that, in our treatment, the second solution $\psi_{n}^{(2)}(x)$ in (23) ceases to exist formally as $a \rightarrow 1$ because of the normalization factor $N^{(2)}$.

In order to see the physical consequence of the caustics anomaly, let us consider an initial state $\psi\left(x, t_{i}\right)$ whose density $\rho_{i}(x)=\left|\psi\left(x, t_{i}\right)\right|^{2}$ has a support only on $\mathbb{R}_{+}$. The state evolves according to the rule set by the kernel (25), and hence the profile will broaden and enter in $\mathbb{R}_{-}$at some later time. The salient feature of the usual quantum caustics observed for quadratic systems is that, at $t_{f}=t_{i}+T$ with $T=($ period of caustics $) \times$ integer, the initial profile is reproduced completely. In our system, however, this is no longer true because for $T=k \pi / \omega$ we have the final state $\psi\left(x, t_{f}\right)=\int d x^{\prime} K\left(x, t_{f} ; x^{\prime}, t_{i}\right) \psi\left(x^{\prime}, t_{i}\right)$ with the density,

$$
\rho_{f}(x)=\left|\psi\left(x, t_{f}\right)\right|^{2}=\cos ^{2}(a k \pi) \rho_{i}(x)+\sin ^{2}(a k \pi) \rho_{i}(-x) .
$$

This shows that, at any later periods, the profile on $\mathbb{R}_{+}$is copied as a mirror image on $\mathbb{R}_{-}$(see Figure 1 ). In particular, when $a=3 / 4$ (i.e., $g=5 \hbar^{2} /(32 m)$ ), the mirror image becomes exactly of the same size as the original for odd $k$, while for even $k$ the 
complete profile is reproduced on $\mathbb{R}_{-}$and $\mathbb{R}_{+}$alternately. We note that this does not contradict the no-cloning theorem [18] because the two 'state spaces' on the half lines, $L^{2}\left(\mathbb{R}_{+}\right)$and $L^{2}\left(\mathbb{R}_{-}\right)$, do not comprise the entire Hilbert space by their direct product, $\mathcal{H}=L^{2}(\mathbb{R} \backslash\{0\}) \neq L^{2}\left(\mathbb{R}_{+}\right) \otimes L^{2}\left(\mathbb{R}_{-}\right)$. In short, rather than making a replica of an arbitrary state prohibited by the no-cloning theorem, the above copying process duplicates a profile by the mirror image.

Since the system discussed in this paper arises in various branches of physics, we expect that our result will find several other applications, and to conclude we just mention a few. First, if one is to confine a particle with more than one channels among which the probability can flow like in certain nuclear states [19] or nano-devices with spin channels, then our quantizations may be adequate to apply. The second is the analysis of black holes, where our system (with and without the harmonic term) describes a particle probing the near-horizon geometry [20, 21, 22]. Further, a straightforward extension of the quantizations of the $n$-body Calogero model (and its related solvable models) along the line outlined here would also enlarge the scope of the application of the model on account of the quantum tunneling now allowed.

Acknowledgement: I.T. is indebted to T. Cheon and R. Sasaki for useful comments. This work has been supported in part by the Grant-in-Aid for Scientific Research (Nos. 10640301 and 13135206) by the Japanese Ministry of Education, Science, Sports and Culture.

\section{Appendix}

In this Appendix we calculate the Feynman kernel $K\left(x_{f}, t_{f} ; x_{i}, t_{i}\right)$ from the energy eigenfunctions (23). Putting $T=t_{f}-t_{i}$ it is given by

$$
K\left(x_{f}, t_{f} ; x_{i}, t_{i}\right)=\left\langle x_{f}\left|e^{-i \widehat{H} T / \hbar}\right| x_{i}\right\rangle=S^{(1)}+S^{(2)},
$$

with

$$
S^{(s)}=\sum_{n=0}^{\infty} \psi_{n}^{(s)}\left(x_{f}\right) e^{-\frac{i}{\hbar} E_{n}^{(s)} T}\left(\psi_{n}^{(s)}\left(x_{i}\right)\right)^{*}, \quad s=1,2 .
$$

To evaluate $S^{(1)}$, we plug (23) into (A.2) using dimensionless variables $y_{i}=\sqrt{m \omega / \hbar} x_{i}$, $y_{f}=\sqrt{m \omega / \hbar} x_{f}$ to find

$$
\begin{aligned}
S^{(1)}= & \sqrt{\frac{m \omega}{\hbar}}\left(\left|y_{f} y_{i}\right|\right)^{c_{1}-1 / 2} e^{-\frac{1}{2}\left(y_{f}^{2}+y_{i}^{2}\right)}\left[\Theta\left(y_{f} y_{i}\right)-\Theta\left(-y_{f} y_{i}\right)\right] \\
& \times \sum_{n=0}^{\infty} e^{-i\left(2 n+c_{1}\right) \omega T} \frac{n !}{\Gamma\left(c_{1}+n\right)} L_{n}^{\left(c_{1}-1\right)}\left(y_{f}^{2}\right) L_{n}^{\left(c_{1}-1\right)}\left(y_{i}^{2}\right),
\end{aligned}
$$


where we have used the relation between the confluent hypergeometric functions and the (associated) Laguerre polynomials,

$$
F(-n, \gamma ; z)=\frac{\Gamma(\gamma) n !}{\Gamma(\gamma+n)} L_{n}^{(\gamma-1)}(z)
$$

Employing the standard trick $T \rightarrow T(1-i \epsilon)$ with an infinitesimal $\epsilon$ in (A.3) to ensure the convergence of the kernel, and using the Hill-Hardy formula (see p.189, ref.[23]),

$$
\begin{aligned}
& \sum_{n=0}^{\infty} w^{n} \frac{n !}{\Gamma(\nu+n+1)} L_{n}^{(\nu)}(u) L_{n}^{(\nu)}(v) \\
& \quad=\left(\frac{1}{1-w}\right) \exp \left(-w \frac{u+v}{1-w}\right)(u v w)^{-\nu / 2} I_{\nu}\left(2 \frac{(u v w)^{1 / 2}}{1-w}\right)
\end{aligned}
$$

valid for $|w|<1$, where $I_{\nu}(z)$ denotes the first kind of the modified Bessel function, we obtain

$$
\begin{aligned}
S^{(1)}=\lim _{\epsilon \rightarrow+0} & \sqrt{\frac{m \omega}{\hbar}}\left(\left|y_{f} y_{i}\right|\right)^{c_{1}-1 / 2} e^{-\frac{1}{2}\left(y_{f}^{2}+y_{i}^{2}\right)}\left[\Theta\left(y_{f} y_{i}\right)-\Theta\left(-y_{f} y_{i}\right)\right] e^{-i \omega T c_{1}} \\
& \times \frac{e^{c_{1} \epsilon / 2}}{1-e^{-i 2 \omega T-\epsilon}} \exp \left(-e^{-i 2 \omega T-\epsilon} \frac{y_{f}^{2}+y_{i}^{2}}{1-e^{-i 2 \omega T-\epsilon}}\right) \\
& \times\left(y_{f}^{2} y_{i}^{2} e^{-i 2 \omega T-\epsilon}\right)^{-\left(c_{1}-1\right) / 2} I_{c_{1}-1}\left(2 \frac{\left(y_{f}^{2} y_{i}^{2} e^{-i 2 \omega T-\epsilon}\right)^{1 / 2}}{1-e^{-i 2 \omega T-\epsilon}}\right),
\end{aligned}
$$

where we have renamed $2 \omega T \epsilon$ as $\epsilon$ for brevity. For $T \neq k \pi / \omega$ with $k=0,1,2, \ldots$, we can take the limit $\epsilon \rightarrow+0$ safely to get

$$
\begin{aligned}
\left.S^{(1)}\right|_{T \neq k \pi / \omega}=\sqrt{\frac{m \omega}{\hbar}} & \left(\left|y_{f} y_{i}\right|\right)^{1 / 2} \frac{1}{2 i \sin (\omega T)} \exp \left(\frac{i}{2} \frac{\cos (\omega T)}{\sin (\omega T)}\left(y_{f}^{2}+y_{i}^{2}\right)\right) \\
& \times\left[\Theta\left(y_{f} y_{i}\right)-\Theta\left(-y_{f} y_{i}\right)\right] I_{c_{1}-1}\left(\frac{\left|y_{f} y_{i}\right|}{i \sin (\omega T)}\right) .
\end{aligned}
$$

The contribution $S^{(2)}$ can be evaluated analogously and the result is exactly the same as $S^{(1)}$ except that $c_{1}$ is now replaced by $c_{2}$ and the factor $\left[\Theta\left(y_{f} y_{i}\right)-\Theta\left(-y_{f} y_{i}\right)\right]$ is removed in (A.6) or (A.7). Combining the two, for $T \neq k \pi / \omega$ we obtain the kernel (25).

On the other hand, for $T=k \pi / \omega$ the kernel can be evaluated directly from (A.1). From the energies (A.1) and the parity $\psi_{n}^{(s)}(-x)=(-1)^{s} \psi_{n}^{(s)}(x)$ of the eigenstates $(23)$, 
we find

$$
\begin{aligned}
K\left(x_{f}, t_{f} ; x_{i}, t_{i}\right)= & \sum_{s=1,2} e^{-i c_{s} k \pi} \sum_{n=0}^{\infty} \psi_{n}^{(s)}\left(x_{f}\right)\left(\psi_{n}^{(s)}\left(x_{i}\right)\right)^{*} \\
= & \frac{1}{2}\left(e^{-i c_{1} k \pi}+e^{-i c_{2} k \pi}\right) \sum_{s=1,2} \sum_{n=0}^{\infty} \psi_{n}^{(s)}\left(x_{f}\right)\left(\psi_{n}^{(s)}\left(x_{i}\right)\right)^{*} \\
& -\frac{1}{2}\left(e^{-i c_{1} k \pi}-e^{-i c_{2} k \pi}\right) \sum_{s=1,2} \sum_{n=0}^{\infty} \psi_{n}^{(s)}\left(-x_{f}\right)\left(\psi_{n}^{(s)}\left(x_{i}\right)\right)^{*} .
\end{aligned}
$$

Using the completeness of the eigenstates and the relations $c_{1}=1+a, c_{2}=1-a$, we obtain (26). 


\section{References}

[1] L.S. Schulman, in "Functional Integration and its Applications", A.M. Arthurs, ed., Clarendon Press, Oxford, 1975.

[2] L.S. Schulman, "Techniques and Applications of Path Integration", John Wiley and Sons, 1981.

[3] B.K. Cheng, Phys. Lett. 101A (1984) 464.

[4] K. Horie, H. Miyazaki and I. Tsutsui, Ann. Phys. 279 (2000) 104.

[5] K. Horie, H. Miyazaki and I. Tsutsui, Phys. Lett. A253 (1999) 259.

[6] F. Calogero, J. Math. Phys. 10 (1969) 2191, 2197; 12 (1971) 419.

[7] B.K. Cheng and F.T. Chan, J. Phys. A20 (1987) 3771.

[8] M. Reed and B. Simon, "Methods of Modern Mathematical Physics II, Fourier analysis, self-adjointness", Academic Press, New York, 1975.

[9] N.I. Akhiezer and I.M. Glazman, "Theory of Linear Operators in Hilbert Space", Vol.II, Pitman Advanced Publishing Program, Boston, 1981.

[10] S. Albeverio, F. Gesztesy, R. Høegh-Krohn and H. Holden, "Solvable Models in Quantum Mechanics", Springer, New York, 1988.

[11] T. Cheon, T. Fülöp and I. Tsutsui, Ann. Phys. 294 (2001) 1.

[12] L. Lathouwers, J. Math. Phys. 16 (1975) 1393.

[13] F. Rellich, Math. Ann. 122 (1951) 343.

[14] A.M. Krall, J. Differential Equations 45 (1982) 128.

[15] I. Tsutsui, T. Cheon and T. Fülöp, Connection Conditions and the Spectral Family under Singular Potentials, in preparation.

[16] I. Tsutsui, T. Fülöp and T. Cheon, J. Math. Phys. 42 (2001) 5687.

[17] D. Peak and A. Inomata, J. Math. Phys. 10 (1969) 1422.

[18] W.K. Wootters and W.H. Zurek, Nature 299 (1982) 802.

[19] Y. Akaishi and T. Yamazaki, Nuclear $\bar{K}$ Bound States in Light Nuclei, KEK Preprint 2001-48, Phys. Rev. C, to appear.

[20] T. Govindarajan, V. Suneeta and S. Vaidya, Nucl. Phys. B583 (2000) 291. 
[21] D. Birmingham, K. Gupta and S. Sen, Phys. Lett. B505 (2001) 191.

[22] S. Mignemi, Mod. Phys. Lett. A16 (2001) 1997.

[23] A. Erdelyi, ed., "Higher Transcendental Functions" Vol.II, McGraw-Hill, New York, 1953. 